\title{
New wrestling rules of anti-inflammatory transrepression by oxysterol receptor LXR revealed
}

\author{
Eckardt Treuter ${ }^{1}$ \\ ${ }^{1}$ Center for Biosciences, Department of Biosciences and Nutrition, Karolinska Institutet, S-14183 Stockholm, Sweden \\ Cell Research (2011) 21:711-714. doi:10.1038/cr.2011.52; published online 29 March 2011
}

While nuclear receptors (NRs) generally function as transcriptional regulators of diverse metabolic, developmental and proliferative processes, some NRs also appear to modulate inflammatory processes such as those directed by Toll-like receptor (TLR) signaling in macrophages and other cell types of the innate immune system $[1,2]$. The medical and pharmaceutical interest in these pathways is considerable since NRs are valid drug targets and inflammatory processes have emerged as intrinsic components of metabolic diseases such as obesity, diabetes and atherosclerosis. Initial research, aimed at understanding the immunosuppressive functions of clinically applied glucocorticoids, had established the importance of negative transcriptional crosstalk between the glucocorticoid receptor (GR) and proinflammatory transcription factors (e.g., NF-kB, AP-1, IRF3). Subsequent investigations revealed intriguing integrative roles of lipid-activated receptors PPARs

\footnotetext{
Correspondence: Eckardt Treuter

Tel: +46-8-52481060

E-mail: eckardt.treuter@ki.se

Abbreviations: liver X receptor (LXR); SUMO2/3 (SU); coronin 2A (CORO2A); SUMO/ sentrin-specific peptidase 3 (SENP3); Calcium/ calmodulin-dependent protein kinase II (CaM$\mathrm{KII}$ ); nuclear receptor corepressor ( $\mathrm{NCoR}$ ); histone deacetylase (HDAC); transducin $\beta 1$ related protein 1 (TBLR1); nuclear receptor coactivator (NCoA); lipopolysaccharide (LPS); Toll-like receptor (TLR); activator protein 1 (AP-1); nuclear factor $\kappa \mathrm{B}(\mathrm{NF}-\kappa \mathrm{B})$; RNA polymerase II (POL II).
}

(peroxisome proliferator-activated receptors, fatty acid ligands) and LXRs (liver X receptors, oxysterols ligands) in controlling both lipid homeostasis and inflammatory processes [3-6]. In particular, anti-inflammatory signaling by these NRs has attracted attention as it provides clues for how metabolic conditions including disease-associated alterations thereof, dietary challenges, environmental cues, and pharmacological interventions, modulate inflammatory processes (and vice versa).

These studies, primarily focusing on the role of PPAR $\gamma$ and LXRs in inhibiting TLR-dependent primary response genes (e.g., inducible nitric oxide synthase $\operatorname{Nos} 2$; interleukin $1 \beta$, $I l 1 b)$ in mouse macrophages, have identified key molecular components of anti-inflammatory crosstalk, referred to as transrepression [1]. Crucial was the recognition that these NRs require posttranslational modification by the family of small ubiquitin-related modifiers (SUMOs) to enter the transrepression pathway. Specifically, ligand activation promotes conjugation of SUMO-1 to PPAR $\gamma$, involving PIAS E3 ligases, or of SUMO-2/3 to LXRs, involving HDAC4 [3, 4]. In both cases, SUMO acceptor lysines are located within the ligand-binding domains. SUMOylated PPAR $\gamma$ or LXRs dock to a conserved corepressor complex at the promoters of inflammatory genes. This "NR corepressor" (NCoR) complex consists of the core subunits NCoR (and/or
SMRT), TBLR1 (and/or TBL1), GPS2 and HDAC3 [7], and apparently plays dual roles in repressing both NR and inflammatory signaling pathways [1-4, $8,9]$. Similarly important was the demonstration that docking of SUMOylated PPAR $\gamma$ or LXRs inhibits the release of the corepressor complex upon inflammatory signaling (i.e., derepression), resulting in maintenance of the repressed state (i.e., transrepression). These findings provided important insights into NR transrepression mechanisms, as they explained how activating ligands (e.g., synthetic LXR agonists) could trigger either metabolic activation or antiinflammatory transrepression. What remained enigmatic was the precise role of NR-SUMOylation in recognizing and docking to the corepressor complex, the further mechanisms of how NR docking would prevent the dismissal of the corepressor complex, and finally whether different signaling inputs (i.e., ligands, post-translational modifications) would perhaps modulate SUMOylation and thus "transrepression capacity" of the respective NRs [10].

A recent study by Chris Glass and colleagues published in Nature [11] has now addressed these key issues by further dissecting LXR transrepression of lipopolysaccharide (LPS)induced TLR4 target genes in mouse macrophages (Figure 1). Huang et al. searched for potential SUMO-interaction motifs (SIMs) in NCoR-associated proteins and identified a motif in the 
C-terminus of coronin 2A (CORO2A), a putative corepressor complex subunit with unknown function [7]. The notion that the CORO2A SIM resembled the SUMO-2/3-selective SIM of the corepressor CoREST1 [12] suggested particular relevance for LXRs, which appear preferentially modified by SUMO-2/3. A series of experimental approaches, including chromatin-immunoprecipitation (ChIP) assays and RNA interference-mediated knockdown, confirmed the specific interactions of CORO2A with SUMOylated LXR $\beta$ in vitro and at the inflammatory Nos 2 and $I l l b$ promoters upon LPS activation. Therefore, CORO2A qualifies as SUMO sensor that mediates docking of SUMOylated LXR $\beta$ to the NCoR complex in macrophages.

Huang et al. additionally provide new insights into the mechanisms of coregulator exchange at inflammatory promoters and into the role of LXRdocking in preventing corepressor dismissal. An initial unexpected observation was that knockdown of CORO2A abolished LPS-induced gene expression and prevented $\mathrm{NCoR}$ dismissal from the promoters upon LPS stimulation. So how does $\mathrm{CORO} 2 \mathrm{~A}$ trigger coregulator exchange? CORO2A belongs to the coronin family of evolutionary conserved actin-binding proteins [13] that contain a $\mathrm{N}$-terminal basic signature motif involved in binding to actin, a central WD40-repeat domain forming a sevenbladed $\beta$-propeller, and a $\mathrm{C}$-terminal coiled-coiled region. Whereas most coronins function in actin-polymerization linked to the cytoskeleton, CORO2A is a nuclear protein $[7,11]$. Nuclear

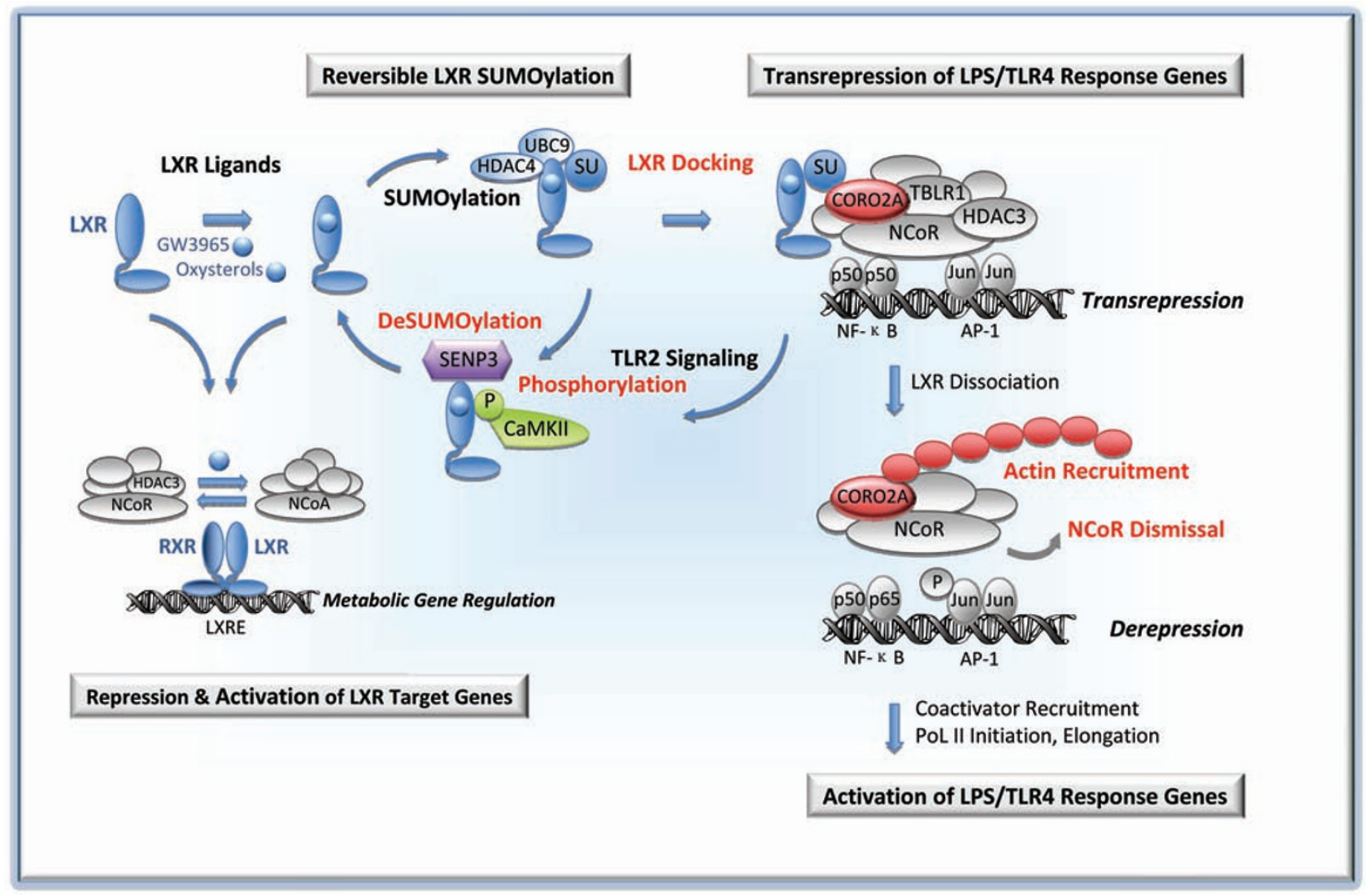

Figure 1 A coronin 2A-dependent LXR transrepression pathway in macrophages. LXR ligands can trigger either conventional activation of direct LXR target genes (e.g., Abca1, Abcg1) or alternative SUMOylation-dependent transrepression of LPS/TLR4 response genes (e.g., Nos2, I/1b, Cc/2). LXRs are specifically modified by SUMO-2/3 (SU) at the ligand-binding domain, which requires the common E2-conjugating enzyme UBC9 and the putative SUMO E3 ligase HDAC4. SUMOylated LXR enters the transrepression pathway by docking to the NCoR corepressor complex at the promoters of LPS/TLR4 response genes. Docking occurs via specific binding of SU to the C-terminal SIM of CORO2A, thereby preventing actindependent derepression and activation steps of inflammatory response genes. Transrepressive LXR can be "inactivated" by deSUMOylation, which requires a sequence of events that are initiated by activation of CaMKII (for example via TLR2 signaling), causing LXR phosphorylation and subsequent recruitment of the SUMO protease SENP3. LXR SUMOylation and the connected transrepression pathway are thus reversible and dynamically regulated in a signal-dependent manner. Highlighted (in red) are the uncovered new aspects of the transrepression pathway in mouse macrophages with an emphasis on the LXR $\beta$ subtype. 
actin, in conjunction with nuclear myosin (NM1) and diverse actin-binding proteins, emerges as a key player in processes responsible for transcriptional initiation and elongation [14]. Regarding the latter it is notable that LPSinduction of primary response genes, which are silenced by NCoR complexes in unstimulated macrophages, seems to occur via transcriptional elongation of a pre-assembled RNA polymerase II $[2,9]$.

Conceivably, Huang et al. considered functional links of CORO2 to nuclear actin. Indeed, the analysis of actinbinding deficient CORO2A mutants, ChIP analysis of actin recruitment, and the use of latrunculin A, which inhibits actin polymerization, validated the cooperative requirement of $\mathrm{CORO} 2 \mathrm{~A}$ and actin for inflammatory derepression. Actin recruitment to inflammatory promoters was dependent on both NCoR and phosphorylation of the c-Jun component of AP-1, further emphasizing its role in coregulator exchange. Most notably, the demonstration that the synthetic LXR agonist GW3965 blocked actin recruitment indicated that SUMOylated LXR via docking to CORO2A somehow interferes with the cooperative exchange function of the CORO2A/actin complex.

Emerging new questions to be answered concern the selectivity of the CORO2A-dependent transrepression mechanism. For example, the SUMO$2 / 3$ specificity of CORO2A suggests that NRs that are modified by SUMO-1, most notably PPAR $\gamma[3,4]$, would not be able to dock to CORO2A and thus utilize alternative mechanisms. Obvious differences between the LXR $\alpha$ and $\beta$ subtypes await further clarification, and various NRs that can be modified by SUMO- 1 or $2 / 3$ [10] potentially trigger CORO2A-dependent derepression mechanisms in other contexts. Yet another aspect concerns the role of CORO2A for SMRT-dependent LPS target genes that can be inhibited by LXRs, since biochemical data suggested copurification of CORO2A with $\mathrm{NCoR}$ but not with SMRT [7]. Regarding the docking mechanism, it remains enigmatic how CORO2A would distinguish SUMOylated LXR from any other SUMOylated proteins or even free SUMOs. It is therefore tempting to speculate that docking might additionally require the specific recognition of the receptor component, for example via NR-binding corepressor complex subunits or modulators (e.g., NCoR, GPS2, HDAC4).

In this respect it is of interest that apparently related transrepression mechanisms allow LXR $\beta$ and LRH-1 (an adopted orphan receptor that binds phospholipids and synthetic ligands) to control the inflammatory "acute phase response" in human and mouse liver hepatocytes [8]. Although the involvement of CORO2A was not addressed in these studies, the subunit GPS2 appeared to establish a physical link between SUMOylated LRH-1 or LXR $\beta$ and the NCoR corepressor complex. Conversely, Huang et al. found that GPS2 was not recruited to CORO2A-dependent TLR4-target promoters in mouse macrophages. Notably, the putative SIMs of GPS2, CORO2A and CoREST1 are all part of coiled-coil regions $[8,11$, 12], suggesting functional relevance and conservation. Thus, GPS2 and CORO2A may function independently or cooperatively in a context-dependent manner.

Another intriguing yet poorly understood aspect concerns the dynamic regulation and reversibility of LXR transrepression, particularly in vivo under chronic inflammatory conditions typically associated with metabolic diseases. A previous study by the authors [15] had noted that TLR2 activation, which is activated by signals other than LPS and linked to calcium/calmodulindependent kinase (CaMKII), abolished LXR transrepression of TLR4 signaling. Huang et al. now report that TLR2dependent CaMKII activation causes phosphorylation of LXR $\beta$, which in turn abolishes LXR SUMOylation and thereby transrepression. The obvious consideration of the involvement of SUMO proteases identified SENP3 as the specific LXR SUMO protease in TLR2-activated macrophages. The identification of a CaMKII/SENP3-dependent LXR deSUMOylation pathway is significant as it provides an example for how intracellular signals that mobilize calcium would modulate the antiinflammatory capacity of LXRs. SENP3 is interestingly regulated by oxidative stress via reactive oxygen species, which play key roles in innate immune responses and in atherogenesis. Clearly, the extent to which SUMOylationdependent transrepression by LXRs, and additional NRs, can be modulated in response to metabolic and pathogenic stress associated with diseases and infections, remains an intriguing aspect to be explored.

Glass and coworkers conclude the study with the in vivo validation of key findings using a thioglycollate-induced sterile peritonitis model in mice, in conjunction with in vivo delivery of CORO2A siRNAs to macrophages, and the analysis of LXR knockout mice. Without any doubt, these achievements substantially advance our current understanding and should stimulate future efforts to dissect NR transrepression pathways in vivo. Future approaches may be to develop diverse mouse models that are defective for specific components of the transrepression pathway, including those determining NR SUMOylation (e.g., acceptor lysine mutants) or those responsible for the recognition and interpretation of NR SUMOylation (e.g., CORO2A, GPS2). These models would help in the development of novel pharmacological strategies that aim to selectively modulate transrepression pathways. As the study by Huang et al. already suggests, the combined treatment of transrepression-selective LXR $\beta$ ligands (which induce SUMOylation) with CaMKII inhibitors (which prevent deSUMOylation) should stabilize 
pools of transrepression-competent SUMOylated LXR in vivo. Conceivably, related strategies could have a hitherto undiscovered potential to treat both the metabolic and inflammatory components of metabolic diseases, such as those triggering atherosclerosis. Last but not least, considering that some aspects of LXR biology linked to cholesterol homeostasis appear differentially regulated in rodents and humans, it will be important to validate the key features of the LXR transrepression pathway in human cell types, along with efforts to identify genetic and epigenetic alterations of pathway components in human disease states.

\section{Acknowledgment}

ET acknowledges expert input from his colleagues and was supported by grants from the Center for Biosciences, the Novo Nordisk Foundation, and the Swedish Research Council.

\section{References}

1 Glass CK, Saijo K. Nuclear receptor transrepression pathways that regulate inflammation in macrophages and $\mathrm{T}$ cells. Nat Rev Immunol 2010; 10:365376.

2 Medzhitov R, Horng T. Transcriptional control of the inflammatory response. Nat Rev Immunol 2009; 9:692-703.

3 Ghisletti S, Huang W, Ogawa S, et al. Parallel SUMOylation-dependent pathways mediate gene- and signal-specific transrepression by LXRs and PPARgamma. Mol Cell 2007; 25:57-70.

4 Pascual G, Fong AL, Ogawa S, et al. A SUMOylation-dependent pathway mediates transrepression of inflammatory response genes by PPAR-gamma. Nature 2005; 437:759-763.

5 Joseph SB, Castrillo A, Laffitte BA, Mangelsdorf DJ, Tontonoz P. Reciprocal regulation of inflammation and lipid metabolism by liver $\mathrm{X}$ receptors. Nat Med 2003; 9:213-219.

6 Ricote M, Li AC, Willson TM, Kelly CJ, Glass CK. The peroxisome proliferator-activated receptor-gamma is a negative regulator of macrophage activation. Nature 1998; 391:79-82.

7 Yoon HG, Chan DW, Huang ZQ, et $a l$. Purification and functional characterization of the human N-CoR complex: the roles of HDAC3, TBL1 and TBLR1. EMBO J 2003; 22:1336-1346.

8 Venteclef N, Jakobsson T, Ehrlund A, et al. GPS2-dependent corepressor/ SUMO pathways govern anti-inflam- matory actions of LRH-1 and LXR-beta in the hepatic acute phase response. Genes Dev 2010; 24:381-395.

9 Hargreaves DC, Horng T, Medzhitov R. Control of inducible gene expression by signal-dependent transcriptional elongation. Cell 2009; 138:129-145.

10 Treuter E, Venteclef N. Transcriptional control of metabolic and inflammatory pathways by nuclear receptor SUMOylation. Biochim Biophys Acta $2010 \mathrm{Dec}$ 21; doi:10.1016/j.bbadis.2010.12.008

11 Huang W, Ghisletti S, Saijo K, et al. Coronin 2A mediates actin-dependent de-repression of inflammatory response genes. Nature 2011; 470:414-418.

12 Ouyang J, Shi Y, Valin A, Xuan Y, Gill G. Direct binding of CoREST1 to SUMO-2/3 contributes to genespecific repression by the LSD1/CoREST1/HDAC complex. Mol Cell 2009; 34:145-154.

13 Uetrecht AC, Bear JE. Coronins: the return of the crown. Trends Cell Biol 2006; 16:421-426.

14 Visa N, Percipalle P. Nuclear functions of actin. Cold Spring Harb Perspect Biol 2010; 2:1-13.

15 Huang W, Ghisletti S, Perissi V, Rosenfeld MG, Glass CK. Transcriptional integration of TLR2 and TLR4 signaling at the $\mathrm{NCoR}$ derepression checkpoint Mol Cell 2009; 35:48-57. 